\title{
1 A unifying framework for quantifying and comparing n-dimensional
}

\section{2 hypervolumes}

3

4 Muyang $\mathrm{Lu}^{1,2}$, Kevin Winner ${ }^{1,2}$, Walter Jetz ${ }^{1,2^{*}}$

$5 \quad{ }^{1}$ Ecology and Evolutionary Biology, Yale University, New Haven, CT, USA

$6 \quad{ }^{2}$ Center for Biodiversity and Global Change, Yale University, New Haven, CT, USA

$7 \quad *$ Correspondence: walter.jetz@yale.edu (W.J.)

11 Abstract

12 1. The quantification of Hutchison's n-dimensional hypervolume has enabled substantial

13 progress in community ecology, species niche analysis and beyond. While non-

14 parametric methods for quantifying and comparing hypervolumes are popular, they do

15 not support a partitioning of the different components and drivers of hypervolume

16 variation. Here, we propose as alternative the use of multivariate normal distributions

17 for the assessment and comparison of niche hypervolumes and introduce this as the

18 multivariate-normal hypervolume (MVNH) framework.

192 . The framework provides parametric measures of the size and dissimilarity of niche

20 hypervolumes, each of which can be partitioned into biologically interpretable

21 components. Specifically, We use 1) the determinant of the covariance matrix (i.e. the

22 generalized variance) of a MVNH as a measure of total niche size, which can be

23 partitioned into the components of univariate niche variances and a correlation

24 component; and 2) the Bhattacharyya distance between two MVNHs as a measure of niche dissimilarity, which can be partitioned into the components of Mahalanobis 
26 distance between hypervolume centroids and the determinant ratio which measures hypervolume size difference.

3. We use empirical examples of community- and species-level analysis to demonstrate the new insights provided by these metrics. We show that the newly proposed framework enables us to quantify the relative contributions of different hypervolume components and to identify the drivers of functional diversity and environmental niche variation.

4. Our approach overcomes several operational and computational limitations of nonparametric methods and provides a framework that offers both unification and granularity in the assessment of niche volumes and differences, which has wide implications for understanding niche evolution, niche shifts and expansion during biotic invasions etc.

39 Keywords: hypervolume; environmental niche; functional diversity; generalized variance;

40 Bhattacharyya distance; beta diversity; standardized ellipse area 
1 Introduction

2 The n-dimensional hypervolume is one of the most fundamental (Hutchinson, 1957;

3 Whittaker, Levin, \& Root, 1973; Pulliam, 2000) and commonly used concepts (Díaz et al.,

4 2015; Blonder, 2018; Pironon et al., 2018) in ecology and evolutionary biology. Hutchinson

5 first proposed to describe species' niche as an n-dimensional hypervolume in which a species

6 can survive and reproduce (Hutchinson, 1957). The use of hypervolume was later extended to

7 describe functional space and trait space (Lamanna et al., 2014; Laughlin, 2014; Pigot et al.,

8 2020). The geometric features of n-dimensional hypervolumes (especially their size and

9 dissimilarity) are associated with a wide range of hypotheses and applications in ecology and

10 evolution. For example, the size of a climatic niche hypervolume is hypothesized to drive

11 species diversification rates: in mammals, species with small niches (specialists) have been

12 shown to have higher speciation rates and lower extinction rates than those with large niches

13 (generalists) (Gómez-Rodríguez, Baselga, \& Wiens, 2015; Rolland \& Salamin, 2016). The

14 size of climatic niche hypervolume is also hypothesized to drive the variation of geographic

15 range size (Slatyer, Hirst, \& Sexton, 2013; Saupe et al., 2015; Cardillo, Dinnage, \&

16 McAlister, 2019; Ficetola, Lunghi, \& Manenti, 2020). Similarity between species'

17 environmental niche hypervolumes or functional trait hypervolumes is used to measure niche

18 divergence or niche packing, which is hypothesized to determine species' coexistence and

19 species richness patterns (Serra-Varela et al., 2015; Pigot, Trisos, \& Tobias, 2016; Castro-

20 Insua, Gómez-Rodríguez, Wiens, \& Baselga, 2018; Read et al., 2018). Niche similarity is

21 also used for within-species comparisons such as assessing the impact of climate change

22 (Tayleur et al., 2015; Gómez, Tenorio, Montoya, \& Cadena, 2016; Zurell, Gallien, Graham,

23 \& Zimmermann, 2018) and whether there are niche shifts during biotic invasion (Lauzeral et

24 al., 2011; Early \& Sax, 2014; Guisan, Petitpierre, Broennimann, Daehler, \& Kueffer, 2014;

25 Davies, Hill, McGeoch, \& Clusella-Trullas, 2019). 
27 The quantification of niche volume and similarity has thus become an urgent pursuit for both

28 theoretical investigation and real-world applications. Recently, non-parametric methods, such

29 as kernel-density estimates (Blonder, Lamanna, Violle, \& Enquist, 2014; Blonder et al., 2018;

30 Eckrich, Albeke, Flaherty, Bowyer, \& Ben-David, 2020; Mammola \& Cardoso, 2020),

31 dynamic range boxes (Junker, Kuppler, Bathke, Schreyer, \& Trutschnig, 2016), minimum

32 convex hulls, and support vector machines (Blonder et al., 2018), have been widely favored

33 because of their minimal assumptions around the distribution of data and the existence of

34 well-documented statistical packages supporting their exploration and use. In these methods,

35 an n-dimensional distribution over environmental or trait space is typically converted to a

36 hypervolume with a boundary defined by a particular isopleth (often 95\%) of the distribution.

37 Niche size is calculated as the volume of the enclosed space. Niche similarity of two

38 hypervolumes can be quantified in multiple ways, either with the union and intersection of

39 hypervolumes (Jaccard and Sorensen indices) or with the distances between the two

40 hypervolumes (Loiseau et al., 2017; Mammola, 2019).

42 These non-parametric methods of quantifying hypervolumes have several methodological

43 limitations: 1) niche volumes and similarity measures are sensitive to the parameterization of

44 the underlying distribution and depend additionally on the choice of isopleth (e.g. $95 \%$ or

$4550 \%), 2$ ) computational costs are high when the dimension of variables and sample size are

46 large (Blonder et al., 2014), and most importantly 3) the non-parametric methods generally

47 cannot say anything about the relative importance of the constituent components of the

48 measures (but see Junker et al., 2016; Loiseau et al., 2017). For example, most non-

49 parametric methods do not provide information on the impact of each individual dimension

50 on the size of a hypervolume. For those that do (e.g. dynamic range box; Junker et al., 2016), 
51 the shape information (correlation among variables) on hypervolume size is ignored. Another

52 example is the use of Jaccard/Sorensen indices and distance metrics for hypervolume

53 similarity comparison. Though the two classes of metrics (Jaccard and Sorensen indices,

54 Euclidean distances between niche centroids) are complementary in capturing hypervolume

55 dissimilarity (Mammola, 2019), no analytical link exists between them for comparing their

56 relative importance on overall hypervolume similarity. The Jaccard and Sorensen indices are

57 used to calculate how much hypervolume is shared between the two hypervolumes, which are

58 more informative when two hypervolumes intersect with each other. On the contrary, the

59 Euclidean distance between hypervolume (centroid distance or minimum distance) is more

60 informative when two hypervolumes are disjunct (Mammola, 2019).

62 The prevalence of non-parametric methods in quantifying hypervolumes also hampers the

63 integration of empirical niche studies with niche theories because empirical metrics are

64 disconnected from theoretical derivations. For example, in the theory of limiting similarity,

65 the outcomes of competition exclusion and niche evolution are typically calculated based on

66 competition coefficients derived from one-dimensional utilization curves (MacArthur \&

67 Levins, 1967; Leimar, Sasaki, Doebeli, \& Dieckmann, 2013). However, the testable

68 theoretical predictions derived by these studies rely on quantitative analyses in higher

69 dimensions (Rappoldt \& Hogeweg, 1980), meaning that with the prevalent non-parametric

70 methods, questions such as "Does higher niche similarity among species lead to more

71 competitive exclusion" could only be studied in a limited or qualitative way (D'Andrea \&

72 Ostling, 2016). Although various parametric dissimilarity metrics have been proposed

73 (Morisita, 1959; Pianka, 1974; Lu, Smith, \& Good, 1989), they were largely ignored because

74 of their conceptual disconnection with the prevalent size metrics of hypervolume. Parametric 
75 measures that have clear analytical links to theory and which unify both size and dissimilarity

76 measurements are urgently needed.

78 To fill in this gap, we propose a framework to quantify and partition niche volume and

79 dissimilarity based on the assumption of multivariate normal (MVN) distribution of the niche

80 variables under study and call this the MVN hypervolume or MVNH framework. Different to

81 currently used non-parametric methods, this partitioning framework provides a powerful

82 quantitative assessment of relative contributions of the constituent components in driving

83 total niche variation. We chose the multivariate normal distribution because it is the most

84 widely used assumption for niche assessment and modeling both in theoretical (MacArthur \&

85 Levins, 1967; Rappoldt \& Hogeweg, 1980; Soberon \& Nakamura, 2009; Jiménez, Soberón,

86 Christen, \& Soto, 2019) and empirical work (Swanson et al., 2015; La Sorte, Fink, \&

87 Johnston, 2018), which provides room for integration and synthesis of size and dissimilarity

88 measures (Lu et al., 1989). We show that in the MVN hypervolume framework niche size is

89 derived from the covariance matrix (Soberon \& Nakamura, 2009), and that niche

90 dissimilarity can be quantified from the Bhattacharyya distance (Bhattacharyya, 1946; Lu et

91 al., 1989; Winner et al., 2018). We demonstrate through analytical derivation and empirical

92 examples how the partitioning of the metrics reveals the key drivers of variations in

93 functional diversity and environmental niche.

95 Materials and Methods

96 In our MVNH framework, we propose that a multi-dimensional niche space be described by a

97 multivariate normal distribution with probability density function:

$98 \quad f_{x}(\mathbf{x})=\frac{\exp \left(-\frac{1}{2}(\mathbf{x}-\boldsymbol{\mu})^{\mathrm{T}} \Sigma^{-1}(\mathbf{x}-\mu)\right)}{\sqrt{(2 \pi)^{n}|\Sigma|}}$ 
100 where $\mathbf{x}=\left\{\mathrm{x}_{1}, \ldots, \mathrm{x}_{n}\right\}$ is a vector in the $\mathrm{n}$-dimensional environmental niche space, $\boldsymbol{\mu}=$

$101\left\{\mu_{1}, \ldots, \mu_{n}\right\}$ is the environmental niche centroid, $\Sigma$ is the covariance matrix for the $n$ -

102 dimensional environmental niche, and $|\Sigma|$ denotes the determinant of the covariance matrix.

104 The size of a hypervolume

105 We define the size of a MVN hypervolume as the determinant of the covariance matrix $|\Sigma|$,

106 also called the "generalized variance" (La Sorte et al., 2018). It has a geometric interpretation

107 as the volume of the n-dimensional parallelepiped spanned by the column or row vectors of

108 the covariance matrix. For example, the covariance matrix $\Sigma$ and the generalized variance $|\Sigma|$

109 of a two-dimensional MVNH are:

$110 \sum=\left[\begin{array}{cc}\sigma_{x}{ }^{2} & \rho_{x y} \sigma_{x} \sigma_{y} \\ \rho_{x y} \sigma_{x} \sigma_{y} & \sigma_{y}{ }^{2}\end{array}\right]$,

$111 \quad \ldots(2)$

$112 \quad|\Sigma|=\sigma_{x}^{2}{\sigma_{y}}^{2}\left(1-\rho_{x y}{ }^{2}\right)$

$113 \quad \ldots(3)$

114 where $\sigma_{\mathrm{x}}{ }^{2}$ is the variance of variable $\mathrm{x}, \sigma_{\mathrm{y}}{ }^{2}$ is the variance of variable $\mathrm{y}$, and $\rho_{\mathrm{xy}}$ is the

115 correlation coefficient between the two environmental variables $\mathrm{x}$ and $\mathrm{y}$. When log-

116 transformed, Equation 3 enables us to additively partition the size of a 2-dimensional

117 hypervolume (in terms of the determinant) into three separate components (Fig. 1c): the

118 variance of the first niche axis $\sigma_{\mathrm{x}}{ }^{2}$, the variance of the second niche axis $\sigma_{\mathrm{y}}{ }^{2}$, and a correlation

119 component $\left(1-\rho_{\mathrm{xy}}{ }^{2}\right)$. The correlation component could be seen as a shrinkage factor which

120 shrinks the niche volume by a factor of $\left(1-\rho_{\mathrm{xy}}{ }^{2}\right)$. Thus, when two niche axes are independent,

121 the niche size is just the product of the variances of individual axes $\sigma_{\mathrm{x}}{ }^{2} \sigma_{\mathrm{y}}{ }^{2}$. Similarly, for a

122 three-dimensional hypervolume, the determinant of the covariance matrix is:

$|\Sigma|={\sigma_{x}}^{2}{\sigma_{y}}^{2}{\sigma_{z}}^{2}\left(1-\rho_{x y}{ }^{2}-\rho_{y z}{ }^{2}-\rho_{x z}{ }^{2}+2 \rho_{x y} \rho_{y z} \rho_{x z}\right)$, 
126 of the determinant generalizes easily to higher dimensions: the determinant is just the product

127 of all individual univariate variances and a correlation component.
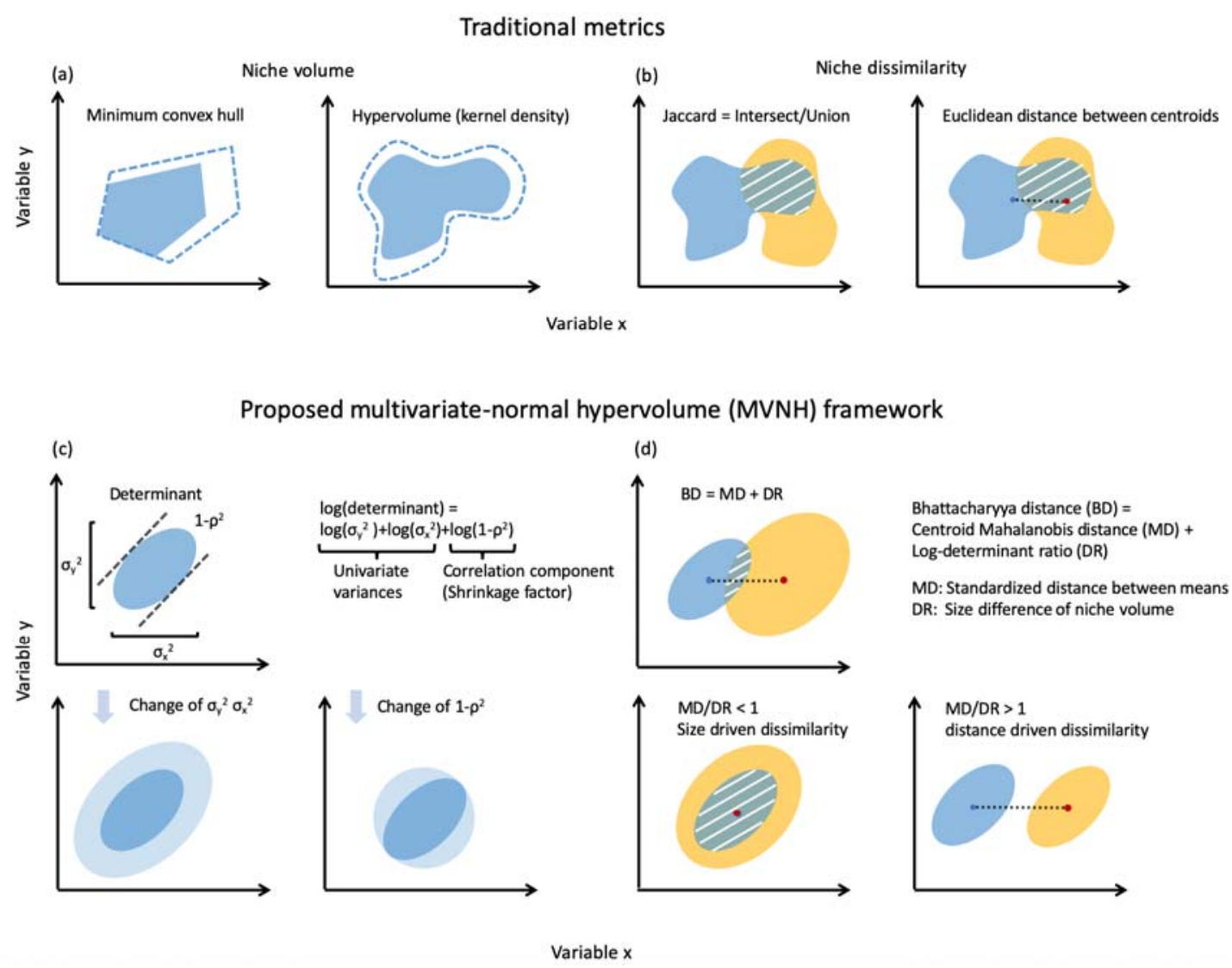

Figure 1. (a) Two commonly used non-parametric niche volume measures: the minimum

130 convex hull and the kernel density estimated hypervolume. The filled area indicates 95\%

131 contour of the hypervolume while the dashed blue lines indicate $90 \%$ contour of the

132 hypervolumes. (b) Two commonly used dissimilarity measures based on non-parametric

133 volume estimates: Jaccard similarity and Euclidean distance between centroids. Orange and

134 blue represent two hypervolumes. White hatches indicate intersecting area between two

135 hypervolumes. (c) The volume of the 2-dimensional niche measured by the determinant of

136 the covariance matrix of environmental variables, which is the product of three components: 
137 two univariate variances $\left(\sigma_{\mathrm{x}}^{2}\right.$ and $\left.\sigma_{\mathrm{y}}{ }^{2}\right)$ and a correlation component which has the effect of

138 shrinking the volume by a factor of $\left(1-\rho^{2}\right)$. Light blue indicates change of hypervolume. (d)

139 Niche dissimilarity of two hypervolumes measured by the Bhattacharyya distance, which is

140 the sum of the Mahalanobis distance between the two niche centroids and a determinant ratio

141 component measuring the differences of the niche volumes. The ratio MD/DR indicates the

142 relative importance of centroid distance and size difference.

144 The determinant also has a close relationship with the standardized ellipse area (SEA, the

145 ellipse with its major and minor axes defined by the principal components; Jackson, Inger,

146 Parnell, \& Bearhop, 2011): it scales with the SEA by a constant ( $\pi$ for two-dimensional niche

147 and $4 \pi / 3$ for three-dimensional niche). Factoring the determinant (as in Equations 3 and 4)

148 can be used to assess the relative importance of the separate components in driving changes

149 in the size of the hypervolume, such as when assessing whether individual trait axis or the

150 constraint on the combination of traits is the major driver of community-wide trait diversity.

152 The dissimilarity of hypervolumes

153 We suggest measuring the dissimilarity of two MVN hypervolumes with the Bhattacharyya

154 distance (Bhattacharyya, 1946; Lu et al., 1989; Minami \& Shimizu, 1999; Fieberg \&

155 Kochanny, 2005; Winner et al., 2018). The Bhattacharyya distance (BD) of two continuous

156 probability distributions, $A$ and $B$ defined on the same domain $\mathcal{X}$ is:

$157 B D(A, B)=-\log B C(A, B)$,

$158 \quad \ldots(5)$

159 where $\mathrm{BC}$ is the Bhattacharyya coefficient:

$$
B C(A, B)=\int_{x \in X} \sqrt{A(x) B(x)} d x
$$


161 Therefore, for two probability distributions, the $\mathrm{BC}$ ranges from 0 to 1 , and the BD ranges

162 from 0 to $\infty$.

163 For two multivariate normal hypervolumes $A$ and $B$, the BD has a closed form:

$$
B D(A, B)=\underbrace{\frac{1}{8}\left(\boldsymbol{\mu}_{A}-\boldsymbol{\mu}_{B}\right)^{T} \overline{\boldsymbol{\Sigma}}^{-1}\left(\boldsymbol{\mu}_{A}-\boldsymbol{\mu}_{B}\right)}_{M D}+\underbrace{\frac{1}{2} \ln \left(\frac{|\overline{\boldsymbol{\Sigma}}|}{\sqrt{\left|\boldsymbol{\Sigma}_{A}\right|\left|\boldsymbol{\Sigma}_{B}\right|}}\right)}_{D R},
$$

166 where $\mu_{\mathrm{A}}$ and $\mu_{\mathrm{B}}$ are the centroids of $\mathrm{A}$ and $\mathrm{B}$ respectively and $\bar{\Sigma}$ is the mean of the

167 covariance matrices of $\mathrm{A}$ and $\mathrm{B}$ :

$168 \bar{\Sigma}=\frac{\Sigma_{A}+\Sigma_{B}}{2}$.

170 The first term in Equation 7 is the Mahalanobis distance (MD) between two hypervolume

171 centroids, and the second term in Equation 7 is the determinant ratio (DR) which measures

172 size difference between A and B (see Fig. 1d). If two hypervolumes have the same size, then

173 the second term in Equation 7 will equal 0. As with Equations 3 and 4, factoring the

174 Bhattacharyya distance according to Equation 7 can be used to assess the relative

175 contributions of the Mahalanobis distance (centroid difference) and the determinant ratio

176 (size difference) to the overall niche dissimilarity. We suggest that the ratio of Mahalanobis

177 distance to determinant ratio (MD/DR), can be used as measure of the relative importance of

178 centroid distance and size difference in driving overall dissimilarity.

180 It should also be noticed that the Mahalanobis distance component in Equations 7 could be

181 linked to an existing partitioning framework which additively partitions the Mahalanobis

182 distance into standardized Euclidean distances along PCA axes which measure the relative

183 importance of each PCA axis (Calenge, Darmon, Basille, Loison, \& Jullien, 2008; Mahony,

184 Cannon, Wang, \& Aitken, 2017). 


\section{Estimators}

187 We used sample means and sample covariances as estimates of $\mu_{\mathrm{A}}, \mu_{\mathrm{B}}, \Sigma_{\mathrm{A}}$, and $\Sigma_{\mathrm{B}}$ for the

188 calculation of the determinant of the covariance matrices and the Bhattacharyya distance with

189 its partitioned components. These plug-in estimates of the Bhattacharyya distance are known

190 to be biased, but the bias is small with low dimensionality (less than 4) of data (Lu et al.,

191 1989; Minami \& Shimizu, 1999). For the purpose of demonstrating the utility of the

192 partitioning framework, our empirical analysis proceeds without applying existing bias-

193 correction methods which have been presented in the literature such as estimating the

194 covariance matrix using Bayesian methods (Swanson et al., 2015), and bias-correction

195 techniques for the Bhattacharyya distance (Minami \& Shimizu, 1999; Winner et al., 2018).

196 Calculation of both metrics only requires a few lines of codes in basic R, which is attached in

197 the supplement (Appendix 1).

199 We also recognize other dissimilarity metrics based on multivariate normal distributions

200 which share a similar form with the Bhattacharyya distance and could be partitioned into a

201 Mahalanobis distance component and a determinant ratio component (see Lu et al., 1989),

202 such as the MacArthur-Levins measure (MacArthur \& Levins, 1967) :

$203 \operatorname{MacArthur}-\operatorname{Levins}(A, B)_{A}=\underbrace{\left(\boldsymbol{\mu}_{A}-\boldsymbol{\mu}_{B}\right)^{T} \overline{\boldsymbol{\Sigma}}^{-1}\left(\boldsymbol{\mu}_{A}-\boldsymbol{\mu}_{B}\right)}_{M D}+\underbrace{\ln \left(\frac{\left|\boldsymbol{\Sigma}_{A}\right|}{\left|\Sigma_{A}\right|\left|\boldsymbol{\Sigma}_{B}\right|}\right)}_{D R}$,

204 MacArthur $-\operatorname{Levins}(A, B)_{B}=\underbrace{\left(\boldsymbol{\mu}_{A}-\boldsymbol{\mu}_{B}\right)^{T} \overline{\boldsymbol{\Sigma}}^{-1}\left(\boldsymbol{\mu}_{A}-\boldsymbol{\mu}_{B}\right)}_{M D}+\underbrace{\ln \left(\frac{\left|\boldsymbol{\Sigma}_{B}\right|}{\left|\Sigma_{A}\right|\left|\boldsymbol{\Sigma}_{B}\right|}\right)}_{D R}$,

206 the Pianka's measure (Pianka, 1974):

$207 \operatorname{Pianka}(A, B)_{A}=\underbrace{\left(\boldsymbol{\mu}_{A}-\boldsymbol{\mu}_{B}\right)^{T} \overline{\boldsymbol{\Sigma}}^{-1}\left(\boldsymbol{\mu}_{A}-\boldsymbol{\mu}_{B}\right)}_{M D}+\underbrace{\frac{1}{2} \ln \left(\frac{|\overline{\boldsymbol{\Sigma}}|}{\sqrt{\left|\boldsymbol{\Sigma}_{A}\right|\left|\boldsymbol{\Sigma}_{B}\right|}}\right)}_{D R}$, 
209 and the Morisita's measure (Morisita, 1959):

$210 \quad$ Morisita $(A, B)=\underbrace{\left(\boldsymbol{\mu}_{A}-\boldsymbol{\mu}_{B}\right)^{T} \overline{\boldsymbol{\Sigma}}^{-1}\left(\boldsymbol{\mu}_{A}-\boldsymbol{\mu}_{B}\right)}_{M D}+\underbrace{\ln \left(\frac{\sqrt{|\overline{\boldsymbol{\Sigma}}|}\left(\sqrt{\left|\boldsymbol{\Sigma}_{A}\right|}+\sqrt{\left|\boldsymbol{\Sigma}_{B}\right|}\right)^{2}}{2 \sqrt{\left|\boldsymbol{\Sigma}_{A}\right|\left|\boldsymbol{\Sigma}_{B}\right|}}\right)}_{D R}$.

$211 \quad \ldots(11)$

212 These metrics differ in their probabilistic interpretations, weighting of each component, and

213 how they calculate the determinant ratio component. We will focus on the use of the

214 Bhattacharyya distance in this paper because it was most studied with known statistical

215 properties (Lu et al., 1989; Minami \& Shimizu, 1999; Winner et al., 2018), and also has a

216 straightforward interpretation as the integration of geometric means of two probability

217 distributions (Equation 6).

220 Empirical examples

221 We will use empirical examples of functional diversity and environmental niche to show the 222 novel insights provided by the partitioning framework especially in quantifying the relative

223 importance of univariate variance and correlation in driving size variation; the relative

224 importance of centroid distance and size difference in driving hypervolume dissimilarity.

225 Community example: Functional diversity and turnover

226 We used a trait dataset of 36 annual plant communities in Western Australia (Data availabile

227 from the Dryad Digital Repository https://doi.org/10.5061/dryad.76kt8 ;Dwyer \& Laughlin,

228 2018) to demonstrate how functional diversity (alpha diversity) and turnover (beta diversity)

229 can be measured in the MVN hypervolume framework using the determinant and the

230 Bhattacharyya distance, respectively. The three measured traits for each species were specific

231 leaf area (SLA), maximum height (MH), and seed mass (SM). We then assessed each 
232 component of the trait volume (SLA variance, MH variance, SM variance and the correlation

233 component) as predicted by growing season available moisture, mean growing season

234 minimum temperature, and soil surface dispersion (dispersion is a measure of heterogeneity)

235 in multiple linear regression. We used the coefficient of variation of each component to

236 assess the relative contribution of each trait volume component to the variation of total trait

237 volume, because the individual niche variances are scalable and not directly comparable with

238 each other. We assessed the environmental drivers of the Bhattacharyya distance (functional

239 turnover) components using multiple regression on distance matrices (MRM) based on a

240 partial Mantel test (Lichstein, 2007).The regression coefficients of MRM are calculated with

241 ordinary least square regression, but the p-values of the coefficients are calculated with

242 matrix permutation tests. The predictor variables were calculated as the Euclidean distances

243 for each environmental variable between sites.

245 Species example: Environmental niche breadth and dissimilarity

246 We studied 260 bird species in the Western Hemisphere with occurrence data extracted from

247 eBird (Sullivan et al., 2009), environmental layers obtained from CHELSA (monthly mean

248 temperature and precipitations for breeding seasons, https://chelsa-climate.org/), and MODIS

249 EVI (https://modis.gsfc.nasa.gov/data/dataprod/mod13.php) at $1 \mathrm{~km}$ resolution to assess the

250 drivers of realized environmental niche breadth (volume) and niche dissimilarity between

251 species. 1000 random points were sampled and spatially thinned to $10 \mathrm{~km}$ distance using the

252 'spThin’ package (Aiello-Lammens, Boria, Radosavljevic, Vilela, \& Anderson, 2015) in R

253 for extraction of environmental data. We then investigated the drivers of environmental niche

254 breadth components using ordinary multiple linear regression, and assessed the drivers of the

255 Bhattacharyya distance components using MRM. We obtained body mass and dietary niche

256 breadth data from the 'EltonTrait' dataset (Wilman et al., 2014). Area and centroid absolute 
257 latitude of geographic range were calculated from IUCN range maps

258 (https://www.iucnredlist.org/resources/spatial-data-download).

260 Comparisons to non-parametric metrics

261 For both the community and niche examples we compared the newly proposed size and

262 dissimilarity metrics from the MVNH framework with measures based on the $95 \%$ isopleth

263 of a box kernel density estimator using the 'hypervolume' package in R (Blonder et al., 2018).

264 For the species environmental niche example, we also compared the determinant of the

265 MVNH with the volume of minimum convex hull (Fig. 1a); for the community functional

266 diversity example, we additionally compared the determinant with three commonly used

267 functional diversity measures: functional divergence, functional dispersion, and functional

268 evenness (Laliberté, Legendre, \& Shipley, 2015). For both examples, we compared the

269 Bhattacharyya distance with Jaccard and Sorensen similarities, centroid distances (Fig. 1b)

270 and minimum distances between hypervolumes.

271

272 Results

273 We use the MVNH framework to firstly analyze drivers of community-level functional

274 diversity and functional turnover, and to secondly assess drivers of species-level

275 environmental niche breadth and dissimilarity. Finally, we show the correlations between the

276 proposed metrics with commonly used non-parametric measures.

278 Community level: Functional diversity

279 We compared functional diversity (measured as the determinant of the trait covariance matrix)

280 and functional turnover (measured as Bhattacharyya distance) among 36 plant communities.

281 For a two-community example (site 1 and site 2), we partitioned the functional diversity of 
282 each site into four components: the univariate variance of each of the three traits (specific leaf

283 area, SLA; maximum height, $\mathrm{MH}$; and seed mass, SM) and the correlation component

284 following Equation 4. Component-wise differences between the two sites add up to the

285 difference in total trait volume (the determinant of the environmental niche covariance matrix)

286 on the log-scale (Fig. 2a).

287 For the 36-community assessment, the coefficient of variation for each trait volume

288 component across the 36 sites was 0.31 for the correlation component, and varied between

$2890.28(\mathrm{MH})$ and $0.16(\mathrm{SM})$ for the individual trait variances. This suggests that the correlation

290 component is the largest driver of the variation in functional diversity among sites. By

291 relating individual trait variances and the correlation component to environmental predictors,

292 we identify mean moisture availability and, less so, minimum temperature as key correlates

293 of trait diversity variation (Fig. 2c, d). The variances of all three traits differ substantially in

294 the strength and direction of their respective associations with environmental predictors.

295 Linear regression shows that none of the examined environmental variables predict the

296 variation of SLA variance (Table 1). Both minimum temperature and moisture availability

297 are positively correlated with maximum height variance, whereas only soil surface dispersion

298 is positively correlated with seed mass variance. Moisture availability is also positively

299 correlated with the correlation component. The overall trait volume (the determinant) is only

300 correlated with moisture availability (Table 1).

301

302

303

304 Table 1. Summary statistics for multiple linear regression of community-level trait volumes,

305 and multiple regression on distance matrices on community-level trait dissimilarity. Specific 
306 leaf area, maximum height, seed mass variances, the correlation component and the

307 determinant are log-transformed. Adjusted $\mathrm{R}^{2}$ is used. Only significant predictors are reported.

\begin{tabular}{lllll}
\hline Response & Predictor & Estimate & $\mathrm{p}$-value & $\mathrm{R}^{2}$ \\
\hline $\begin{array}{l}\text { Specific leaf area } \\
\text { variance }\end{array}$ & -- & - & & -- \\
$\begin{array}{l}\text { Maximum height } \\
\text { variance }\end{array}$ & Minimum temperature & -- & - \\
& Moisture availability & 0.125 & $<0.01$ & 0.19 \\
Seed mass variance & Soil surface dispersion & 0.102 & $<0.05$ & \\
Correlation component & Moisture availability & 0.066 & $<0.05$ & 0.25 \\
\hdashline & Moisture availability & 0.228 & $<0.001$ & 0.37 \\
\hline Determinant & & 0.369 & $<0.001$ & 0.27 \\
\hline & & & & \\
Mahalanobis distance & Distance (moisture availability) & 0.18 & $<0.001$ & 0.20 \\
& Distance (minimum temperature) & -0.07 & $<0.05$ & \\
Determinant ratio & Distance (moisture availability) & 0.08 & $<0.001$ & 0.13 \\
\hdashline Bhattacharyya distance & Distance (moisture availability) & 0.26 & $<0.001$ & 0.18 \\
\hline
\end{tabular}

310 We measured the pairwise functional turnover between any two sites as the Bhattacharyya

311 distance between their MVN hypervolumes, and according to Equation 7, partitioned it into

312 the Mahalanobis distance (which measures the standardized distance between community

313 means) and the determinant ratio (which measures the differences in functional alpha

314 diversity) (see a two-community example in Fig. 2b). The ratio of Mahalanobis distance to

315 determinant ratio (MD/DR) reflects the relative importance of difference in community

316 means and trait diversity differences in driving the overall community-level functional

317 turnover. The median ratio MD/DR across all pairs of sites is 0.63 , suggesting functional

318 turnover among communities is mainly driven by the variation in functional alpha-diversity.

319 The functional turnover is more determined by moisture availability than by minimum

320 temperature (Fig. 2e, f). Multiple regression on distance matrices suggests that the

321 Mahalanobis distance is correlated with both minimum temperature and moisture availability,

322 but the determinant ratio is only correlated with moisture availability (Table 1). 


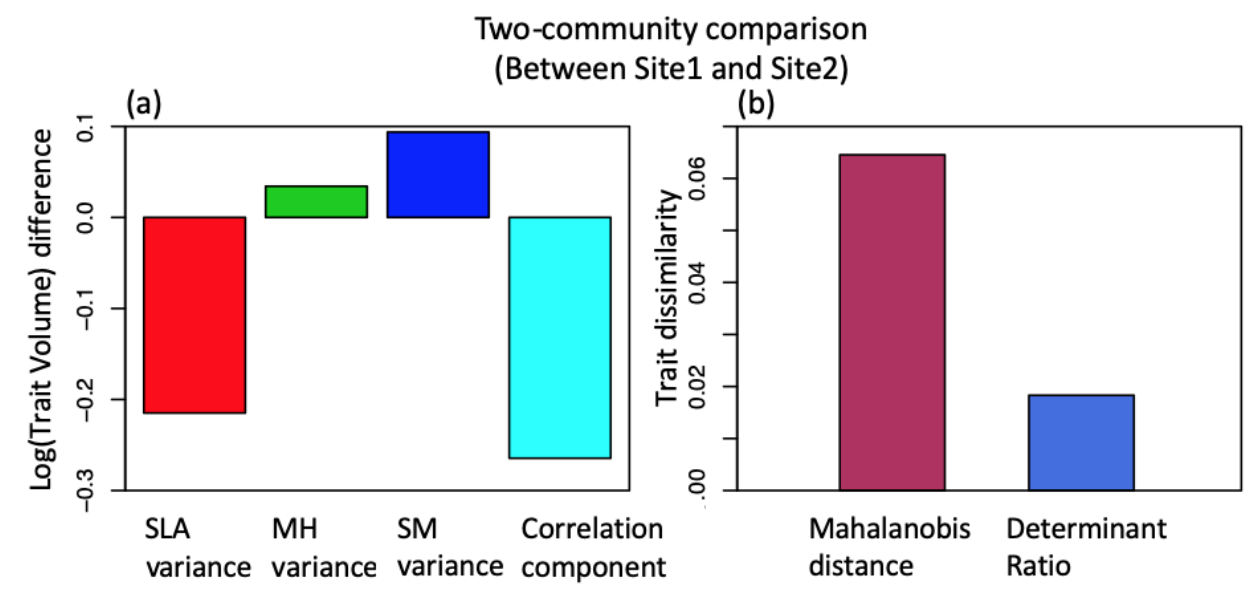

Environmental predictors of community trait variation across 36 sites

(c)

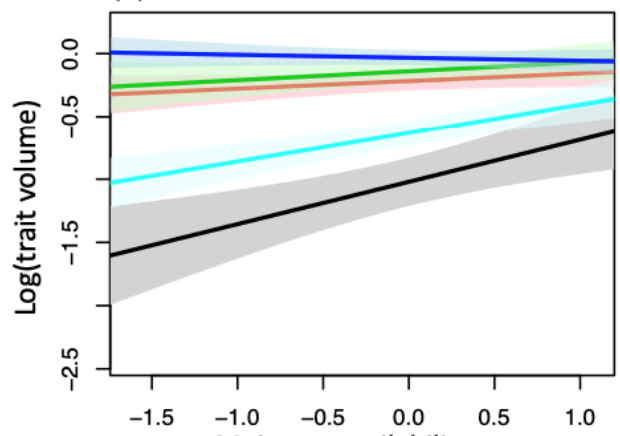

(e)

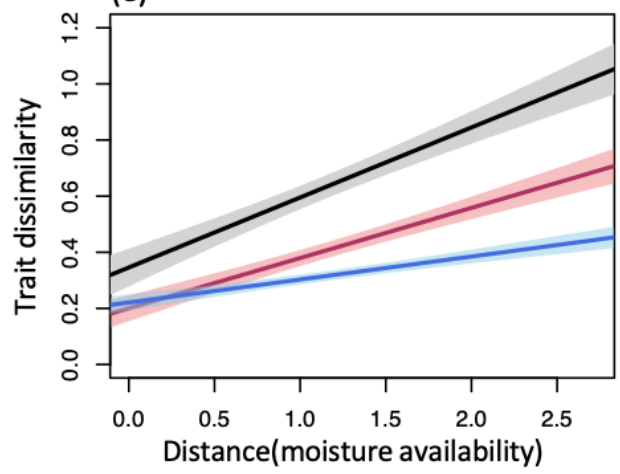

(d)

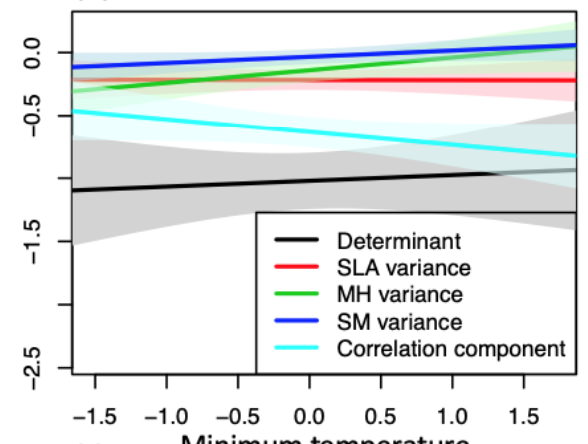

(f) Minimum temperature

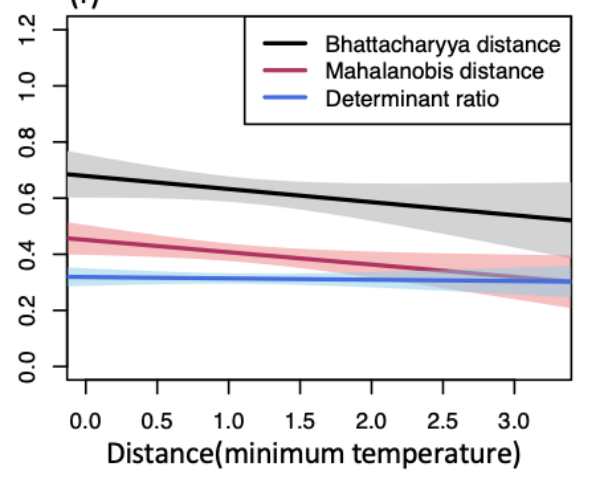

Figure 2. Community-level functional diversity assessment. (a) Component-wise differences

325 between site 1 and site 2 in a) functional trait volume (e.g. functional diversity) and b)

326 Bhattacharyya distance of functional traits. (c)-(f) Change of trait volume and pairwise

327 community-level trait dissimilarity among all 36 sites of annual plant communities with

328 environmental variables in southwest Western Australia. Solid lines are the fitted linear

329 regression. Shaded area represents $95 \%$ confidence interval of linear regression. See table 1

330 for summary statistics. 
332 Species level: environmental niches

333 We used the presented methodology to analyze and compare the three-dimensional

334 environmental niches (temperature, precipitation, and EVI) of 260 bird species in the New

335 World. We partitioned the total environmental niche volume for each single species into the

336 three environmental variable variance components and the correlation component. For a two-

337 species example, we attributed the difference in niche volume between the white-throated

338 swift and the tricolored blackbird to differences in temperature variance, precipitation

339 variance, EVI variance and the correlation component (Fig. 3a). For the 260-species,

340 assessment, we found that the coefficient of variation for each of the niche volume

341 components among 260 bird species was 0.79 for precipitation variance, 0.67 for temperature

342 variance, 0.44 for EVI variance, and 0.22 for the correlation component, suggesting that

343 precipitation niche breadth variation contributes most to the species-level niche volume

344 variation. Range size and centroid latitude are the two main drivers of realized environmental

345 niche volume (Fig. 3c, d), but the predictors for each niche volume component are different:

346 the precipitation variance and the correlation component are only correlated with the centroid

347 latitude while the temperature variance and the EVI variance are correlated with both

348 centroid latitude and range size (Table 2). The median ratio between Mahalanobis distance

349 and determinant ratio is 2.43 , suggesting that the distance between niche centroids is more

350 important than niche volume differences in driving overall environmental niche dissimilarity

351 among the 260 bird species. When the multiple regression on distance matrices (MRM) is

352 applied, different components of the Bhattacharyya distance have different determining

353 factors: the Mahalanobis distance is only correlated with centroid latitude; while the

354 determinant ratio is correlated with both range size and centroid latitude (Table 2). 
356 Table 2. Summary statistics for multiple linear regression of environmental niche volumes,

357 and Multiple regression on distance matrices on environmental niche dissimilarity. Area,

358 temperature, precipitation, EVI variances, the correlation component and the determinant are

$359 \log$-transformed. Adjusted $\mathrm{R}^{2}$ is used. Only significant predictors are reported.

\begin{tabular}{lllll}
\hline response & predictor & estimate & p-value & $\mathrm{R}^{2}$ \\
\hline Temperature variance & Area & 1.13 & $<0.001$ & 0.14 \\
& Centroid latitude & 0.84 & $<0.001$ & \\
Precipitation variance & Centroid latitude & -5.72 & $<0.001$ & 0.69 \\
EVI variance & Area & 0.70 & $<0.001$ & 0.13 \\
& Centroid latitude & -0.46 & $<0.001$ & \\
Correlation component & Centroid latitude & -0.67 & $<0.001$ & 0.22 \\
\hdashline Determinant & Area & 2.09 & $<0.001$ & 0.55 \\
& Centroid latitude & -5.97 & $<0.001$ & \\
\hline \multirow{4}{*}{ Mahalanobis distance } & & & & \\
Determinant ratio & Distance (Centroid latitude) & 32.62 & $<0.001$ & 0.13 \\
& Distance (Area) & -2.66 & $<0.001$ & 0.23 \\
\cline { 1 - 4 } Bhattacharyya distance & Distance (Centroid latitude) & 9.78 & $<0.001$ & \\
\hline
\end{tabular}


Two-species comparison

(a)

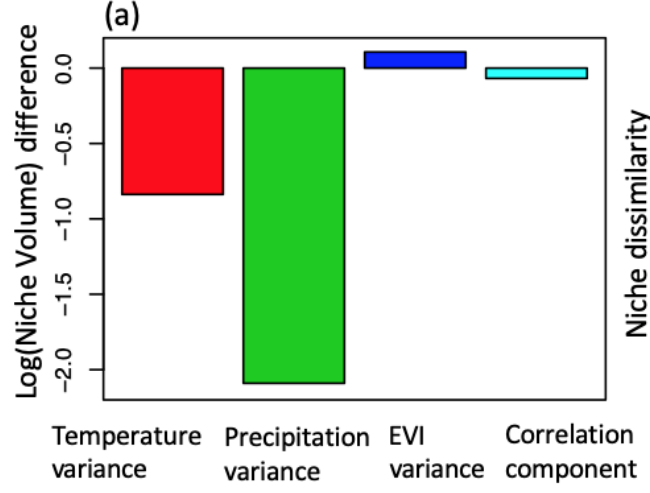

(b)

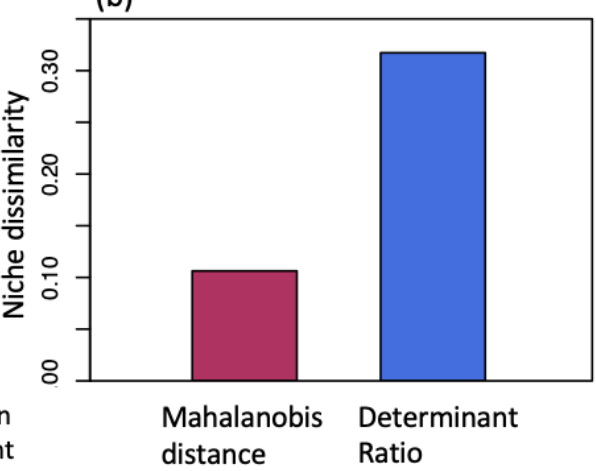

Drivers of environmental niche variation across 260 species

(c)

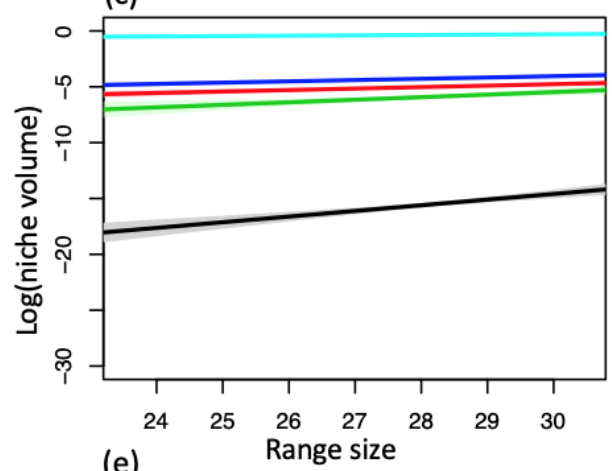

(e)

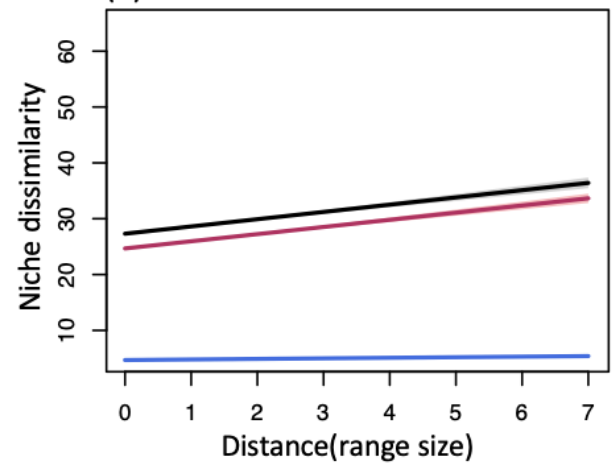

(d)

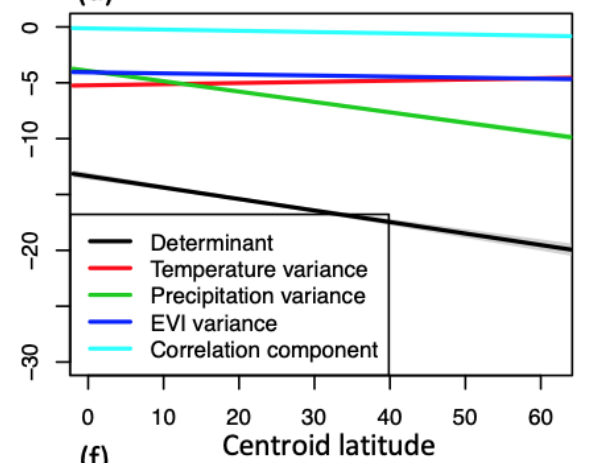

(f)

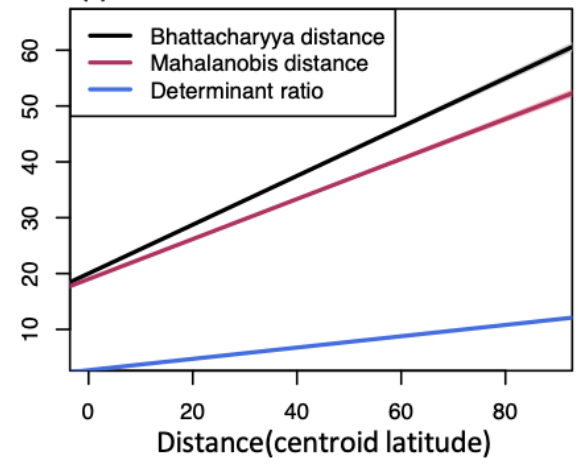

Figure 3. Species-level environmental niche assessment. (a) Component-wise environmental

364 niche volume differences and (b) Bhattacharyya distance of environmental niche dissimilarity

365 between two example species (White-throated Swift and Tricolored Blackbird) (c)-(f)

366 Change of niche volume and pairwise niche dissimilarity among 260 bird species with range

367 size and centroid latitude. Solid lines are the fitted linear regression. Shaded area represents

$36895 \%$ confidence interval of linear regression. See table 2 for summary statistics. 
370 Comparison with other metrics

371 Community-level functional traits: The determinant of trait covariance matrix as the measure

372 of functional diversity had the highest correlation with the area of the $95 \%$ isopleth of a

373 kernel density derived hypervolume (0.89), followed by functional dispersion (0.79) and

374 minimum convex hull (0.65) (additional methods in Fig. 4a). The Bhattacharyya distance had

375 a similar correlation with Euclidean centroid distance (0.70), Jaccard similarity (-0.69) and

376 Sorensen similarity (-0.71). The Mahalanobis distance component was more correlated with

377 Euclidean centroid distance (0.73) than with Jaccard similarity (-0.57) or Sorensen similarity

$378(-0.59)$, while the determinant ratio component had a relatively low correlation with the

379 Euclidean centroid distance (0.42; Fig. 4b). All components were uncorrelated with minimum

380 distance between hypervolumes (Fig. 4b).

381

382 Species-level environmental niche: the determinant of the environmental niche covariance

383 matrix correlates most strongly with the area of the $95 \%$ isopleth of the kernel density

384 derived hypervolume (0.85), followed by the minimum convex hull (0.77, Fig. 4c). The

385 Bhattacharyya distance and its Mahalanobis distance component are most correlated with the

386 Euclidean centroid distance (0.85 and 0.82 respectively); the determinant ratio is similarly

387 correlated with most alternative metrics except for minimum distance between hypervolumes

388 (Fig. 4d). 

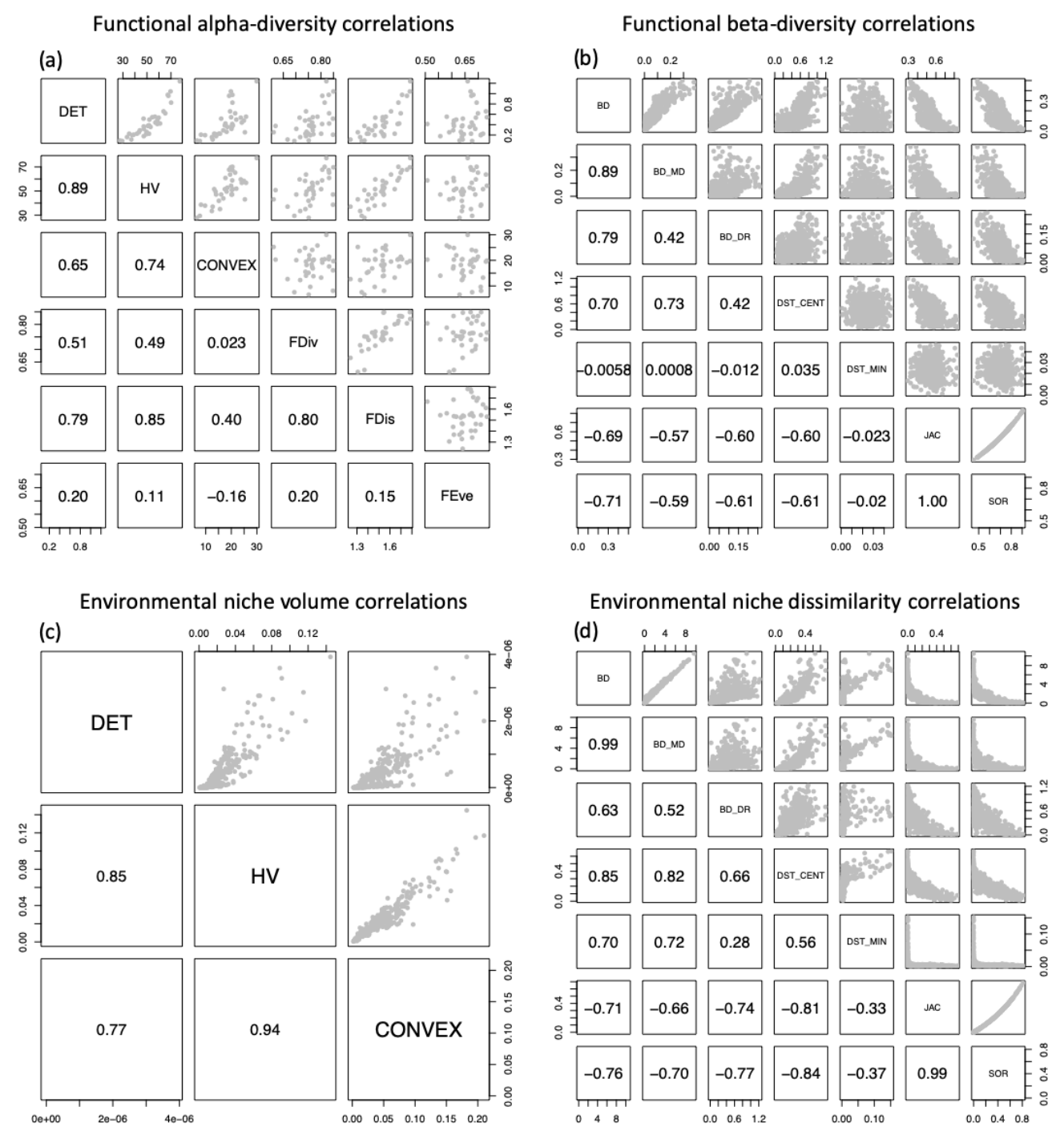

390 Figure 4. (a) Correlations among functional alpha-diversity measures for the 36 sites. (b)

391 Correlations among pairwise functional turnover measures. (c) Correlations among

392 environmental niche volume measures for the 260 bird species. (d) Correlations among

393 pairwise environmental niche dissimilarity measures. 'DET' stands for the determinant of the

394 environmental niche or functional trait covariance matrix, 'HV' for kernel density derived

395 hypervolume, 'CONVEX' for minimum convex hull, 'FDiv' for functional divergence, 'FDis'

396 for functional dispersion, 'FEve' for functional evenness. 'BD' stands for Bhattacharyya

397 distance, 'BD_MD' for the Mahalanobis distance component of BD, 'BD_DR” for the 
398 determinant ratio component of BD, 'DST_CENT' for Euclidean distance between centroids,

399 'DST_MIN' for minimum distance between kernel density derived hypervolumes, 'JAC' for

400 Jaccard similarity, 'SOR' for Sorensen similarity.

401

402 Discussion

403 We proposed a new parametric hypervolume framework based on multivariate normal

404 distributions and corresponding size and dissimilarity metrics. The greatest advantage of the

405 MVN hypervolume approach is the analytical clarity and insights provided by the partitioned

406 components of the niche size and dissimilarity metrics. The MVNH framework is also

407 significantly faster than the widely used KDE approach. For example, using the 'Iris' dataset

408 in $\mathrm{R}$ (a dataset with 150 rows and 3 columns), it takes 6 seconds to compute the hypervolume

409 using KDE but only 0.001 seconds to compute the hypervolume using MVNH. Although the

410 determinant of the covariance matrix has been used as a total niche volume measure in some

411 studies (Soberon \& Nakamura, 2009; La Sorte et al., 2018), it has never been illustrated that

412 the determinant could be interpreted as the product of individual niche variances and a

413 correlation component. The Bhattacharyya distance has been used as a measure of home

414 range overlap in animal movements (Fieberg \& Kochanny, 2005; Winner et al., 2018). We

415 suggest that its use in trait or environmental space (Lu et al., 1989; Minami \& Shimizu, 1999),

416 and in particular the interpretation of the partitioned components, offers valuable biological

417 insights. Specifically, the Mahalanobis distance between hypervolume centroids can be

418 interpreted as the standardized distance between optimal traits of two communities or

419 between the optimal environmental conditions of two species under the assumptions that

420 mean community traits represents the optimal traits under equilibrium (Blonder et al., 2015)

421 and that niche centroid has the highest fitness for a species (Sagarin, Gaines, \& Gaylord,

422 2006). The determinant ratio can be interpreted as the functional diversity difference between 
423 communities or as niche volume differences between species. The Bhattacharyya distance

424 (BD) not only avoids choosing ad hoc algorithm parameters, but more importantly reconciles

425 the limitations of the two commonly used classes of dissimilarity metrics. When Jaccard or

426 Sorensen indices become uninformative for disjunct hypervolumes, the BD incorporates the

427 distance information through the Mahalanobis distance component. When the centroid

428 distance metric becomes uninformative for two close and overlapping hypervolumes, the BD

429 incorporates the shape information through the determinant ratio component.

431 The good concordance between our method and the kernel density method across each of our

432 studies suggests that the determinant as a measure of niche volume is robust to different

433 distributions of data. For niche dissimilarity measures, the moderate amount of correlation

434 between the partitioned components of BD with the Jaccard, Sorensen and the centroid

435 Euclidean distances shows that the partitioned components of BD both capture a part of the

436 shape information of the hypervolume. Therefore, our BD metrics combine the important

437 interpretations of both centroid Euclidean distance metrics and the Jaccard and Sorensen

438 metrics of niche dissimilarity. More importantly, the relative importance of centroid

439 difference and volume difference are directly comparable using the BD metrics.

440 Other metrics such as the MacArthur-Levins measure (Equation 9), the Pianka's measure

441 (Equation 10) and the Morisita's measure (Equation 11) could be similarly partitioned into a

442 Mahalanobis distance component and a determinant ratio component (Lu et al., 1989), though

443 their properties are less well known than the Bhattacharyya distance (Minami \& Shimizu,

444 1999; Winner et al., 2018). The shared Mahalanobis distance component in all these metrics

445 could be further partitioned into standardized Euclidean distance along different PCA axes

446 according to a previously proposed partitioning framework (Calenge et al., 2008; Mahony et 
447 al., 2017), which measures the relative importance of each PCA axis in determining the

448 overall Mahalanobis distance.

450 Case studies

451 Through the partitioning framework, our methods provide a novel understanding of each

452 component driver of community functional diversity or species environmental niche volume.

453 In the functional diversity example we demonstrated that for the 36 annual plant communities

454 the constraints on combination of traits is the major driver of functional alpha diversity

455 variation rather than the change of individual trait variances, which in turn is shaped by

456 moisture availability; the major driver of functional community turnover among is the

457 variation of functional alpha-diversity rather than the mean trait differences between

458 communities. In the species environmental niche example, our method shows that the major

459 driver of niche volume variation is the precipitation niche breadth variation (Fig. 3d) which is

460 determined by centroid latitude of species' range. We also show that the range size-niche

461 breadth relationship of total environmental niche breadth is mainly driven by the range size-

462 temperature niche breadth and range size-EVI niche breadth relationship (Table 2).

\section{Caveats}

465 A common limitation of all hypervolume metrics is the curse of dimensionality and

466 collinearity among axes (Blonder et al., 2014; D’Andrea \& Ostling, 2016). The use of the

467 determinant is especially sensitive to collinearity because any pair of fully dependent

468 columns or rows in a covariance matrix will necessarily result in a determinant of zero.

469 Increasing dimensions of trait or environmental data will increase the chance of collinearity

470 and is also more likely to violate the multivariate normal assumption. Such an issue could be

471 resolved by choosing the biologically relevant variables a priori or using common dimension 
472 reduction techniques applied in non-parametric methods such as PCA (Junker et al., 2016).

473 Because PCA creates orthogonal axes, the correlation component will always equal 1 if PCA

474 is applied, and thus it is only recommended when the correlation among axes is of no interest

475 to the study.

477 Potential

478 The presented size and dissimilarity measures are to our knowledge the first parametric

479 metrics that allow a partitioning of $n$-dimensional hypervolumes. The partitioning of total

480 niche volume and niche dissimilarity enables more rigorous testing of ecological and

481 evolutionary hypotheses beyond those we explored in our two case studies. For example,

482 when investigating the spatial scaling of niche volume (Pearman, Guisan, Broennimann, \&

483 Randin, 2008; Connor et al., 2018), the scaling relationship could be broken down to the

484 scaling of univariate niche variances and correlation component, each of which could be

485 linked to different sets of predictors. When studying niche shifts during biotic invasion

486 (Lauzeral et al., 2011; Hill, Hoffmann, Macfadyen, Umina, \& Elith, 2012; Wiens, Litvinenko,

487 Harris, \& Jezkova, 2019), the BD could be used to determine whether the niche shift is

488 mainly caused by shifts in niche centroids or caused by volume expansion or reduction; the

489 determinant could be used to assess which niche axis is most responsible for the total volume

490 expansion or reduction. In niche evolution studies, the evolution of niche dissimilarity

491 (Nunes \& Pearson, 2017; Castro-Insua et al., 2018) between two species could be traced to

492 contributions by niche breadth or center differences to the total niche divergence. The

493 parametric measures also have closer links to theory as theoretical predictions of niche

494 evolution are usually derived from covariance matrixes with multivariate normal

495 approximations (Lande \& Arnold, 1983; Caetano \& Harmon, 2019). In other words, the

496 newly proposed metrics have the potential to provide more mechanistic understanding of 
497 biodiversity patterns and niche evolution, and to pave the way for more accurate forecasts for

498 biodiversity change.

499

500 Acknowledgement

501 We thank Jetz Lab members for discussion of the work, especially Richard Li, Aurore

502 Maureaud and Ben Carlson for comments on the first draft.

503

504 Authors' contributions

505 ML and WJ conceived the ideas. ML analyzed the data with substantial inputs from KW and

506 WJ. ML led the writing of the manuscript. All authors contributed critically to the drafts and

507 gave final approval for publication.

509 Reference

510 Aiello-Lammens, M. E., Boria, R. A., Radosavljevic, A., Vilela, B., \& Anderson, R. P.

511 (2015). spThin: An R package for spatial thinning of species occurrence records for use

512 in ecological niche models. Ecography, 38(5), 541-545. doi:10.1111/ecog.01132

513 Bhattacharyya, A. (1946). On a Measure of Divergence between Two Multinomial

514 Populations. Sankhyā: The Indian Journal of Statistics. doi:10.1038/157869b0

515 Blonder, B. (2018). Hypervolume concepts in niche- and trait-based ecology. Ecography,

$516 \quad 41(9), 1441-1455$. doi:10.1111/ecog.03187

517 Blonder, B., Lamanna, C., Violle, C., \& Enquist, B. J. (2014). The n-dimensional

518 hypervolume. Global Ecology and Biogeography, 23(5), 595-609.

519 doi:10.1111/geb.12146

520 Blonder, B., Morrow, C. B., Maitner, B., Harris, D. J., Lamanna, C., Violle, C., ... Kerkhoff, 521 A. J. (2018). New approaches for delineating $\mathrm{n} \square$ dimensional hypervolumes. Methods in 
Ecology and Evolution, 9(2), 305-319. doi:10.1111/2041-210X.12865

523 Blonder, B., Nogués-Bravo, D., Borregaard, M. K., Donoghue, J. C., Jørgensen, P. M., Kraft,

524 N. J. B., ... Enquist, B. J. (2015). Linking environmental filtering and disequilibrium to

525 biogeography with a community climate framework. Ecology, 96(4), 972-985.

$526 \quad$ doi:10.1890/14-0589.1

527 Caetano, D. S., \& Harmon, L. J. (2019). Estimating correlated rates of trait evolution with

528 uncertainty. Systematic Biology, 68(3), 412-429. doi:10.1093/sysbio/syy067

529 Calenge, C., Darmon, G., Basille, M., Loison, A., \& Jullien, J. M. (2008). The factorial

530 decomposition of the Mahalanobis distances in habitat selection studies. Ecology, 89(2),

$531 \quad 555-566$. doi:10.1890/06-1750.1

532 Cardillo, M., Dinnage, R., \& McAlister, W. (2019). The relationship between environmental

533 niche breadth and geographic range size across plant species. Journal of Biogeography,

$534 \quad$ 46(1), 97-109. doi:10.1111/jbi.13477

535 Castro-Insua, A., Gómez-Rodríguez, C., Wiens, J. J., \& Baselga, A. (2018). Climatic niche

536 divergence drives patterns of diversification and richness among mammal families.

537 Scientific Reports, 8(1), 8781. doi:10.1038/s41598-018-27068-y

538 Connor, T., Hull, V., Viña, A., Shortridge, A., Tang, Y., Zhang, J., ... Liu, J. (2018). Effects

539 of grain size and niche breadth on species distribution modeling. Ecography, 41(8),

$540 \quad$ 1270-1282. doi:10.1111/ecog.03416

541 D’Andrea, R., \& Ostling, A. (2016). Challenges in linking trait patterns to niche

542 differentiation. Oikos, 125(10), 1369-1385. doi:10.1111/oik.02979

543 Davies, S. J., Hill, M. P., McGeoch, M. A., \& Clusella-Trullas, S. (2019). Niche shift and

544 resource supplementation facilitate an amphibian range expansion. Diversity and

545 Distributions, 25(1), 154-165. doi:10.1111/ddi.12841

546 Díaz, S., Kattge, J., Cornelissen, J. H. C., Wright, I. J., Lavorel, S., Dray, S., ... Gorné, L. D. 
(2015). The global spectrum of plant form and function. Nature, 1-17.

$548 \quad$ doi:10.1038/nature16489

549 Dwyer, J. M., \& Laughlin, D. C. (2018). Data from: Constraints on trait combinations explain

550 climatic drivers of biodiversity: the importance of trait covariance in community

$551 \quad$ assembly. Dryad, Dataset. doi:10.5061/dryad.76kt8

552 Early, R., \& Sax, D. F. (2014). Climatic niche shifts between species' native and naturalized

553 ranges raise concern for ecological forecasts during invasions and climate change.

554 Global Ecology and Biogeography, 23(12), 1356-1365. doi:10.1111/geb.12208

555 Eckrich, C. A., Albeke, S. E., Flaherty, E. A., Bowyer, R. T., \& Ben-David, M. (2020). rKIN:

556 Kernel-based method for estimating isotopic niche size and overlap. Journal of Animal

557 Ecology, 89(3), 757-771. doi:10.1111/1365-2656.13159

558 Ficetola, G. F., Lunghi, E., \& Manenti, R. (2020). Microhabitat analyses support

559 relationships between niche breadth and range size when spatial autocorrelation is strong.

$560 \quad$ Ecography, 724-734. doi:10.1111/ecog.04798

561 Fieberg, J., \& Kochanny, C. O. (2005). Quantifying home-range overlap: The importance of

562 the utilization distribution. Journal of Wildlife Management. doi:10.2193/0022-

$563 \quad 541 \mathrm{X}(2005) 69[1346: \mathrm{QHOTIO}] 2.0 . \mathrm{CO} ; 2$

564 Gómez-Rodríguez, C., Baselga, A., \& Wiens, J. J. (2015). Is diversification rate related to

$565 \quad$ climatic niche width? Global Ecology and Biogeography, 24(4), 383-395.

566 doi:10.1111/geb.12229

567 Gómez, C., Tenorio, E. A., Montoya, P., \& Cadena, C. D. (2016). Niche-tracking migrants

568 and niche-switching residents: evolution of climatic niches in New World warblers

569 (Parulidae). Proceedings of the Royal Society B: Biological Sciences, 283(1824),

20152458. doi:10.1098/rspb.2015.2458

571 Guisan, A., Petitpierre, B., Broennimann, O., Daehler, C., \& Kueffer, C. (2014). Unifying 
572

573

574

575

576

577

578

579

580

581

582

583

584

585

586

587

588

589

590

591

592

593

594

595

596

niche shift studies $\square$ : insights from biological invasions. Trends in Ecology \& Evolution, 29(5), 260-269. doi:10.1016/j.tree.2014.02.009

Hill, M. P., Hoffmann, A. A., Macfadyen, S., Umina, P. A., \& Elith, J. (2012). Understanding niche shifts: using current and historical data to model the invasive redlegged earth mite, Halotydeus destructor. Diversity and Distributions, 18(2), 191-203. doi:10.1111/j.14724642.2011.00844.x

Hutchinson, G. E. (1957). Concluding Remarks. Cold Spring Harbor Symposia on Quantitative Biology, 22, 415-427. doi:10.1101/SQB.1957.022.01.039

Jackson, A. L., Inger, R., Parnell, A. C., \& Bearhop, S. (2011). Comparing isotopic niche widths among and within communities: SIBER - Stable Isotope Bayesian Ellipses in R. Journal of Animal Ecology, 80(3), 595-602. doi:10.1111/j.1365-2656.2011.01806.x

Jiménez, L., Soberón, J., Christen, J. A., \& Soto, D. (2019). On the problem of modeling a fundamental niche from occurrence data. Ecological Modelling, 397(5), 74-83. doi:10.1016/j.ecolmodel.2019.01.020

Junker, R. R., Kuppler, J., Bathke, A. C., Schreyer, M. L., \& Trutschnig, W. (2016). Dynamic range boxes - a robust nonparametric approach to quantify size and overlap of ndimensional hypervolumes. Methods in Ecology and Evolution, (i), 1503-1513. doi:10.1111/2041-210X.12611

La Sorte, F. A., Fink, D., \& Johnston, A. (2018). Seasonal associations with novel climates for North American migratory bird populations. Ecology Letters, 845-856. doi:10.1111/ele.12951

Laliberté, E., Legendre, P., \& Shipley, B. (2015). FD: measuring functional diversity from multiple traits, and other tools for functional ecology. R Package.

Lamanna, C., Blonder, B., Violle, C., Kraft, N. J. B., Sandel, B., Šímová, I., ... Enquist, B. J. (2014). Functional trait space and the latitudinal diversity gradient. Proceedings of the 

doi:10.1073/pnas.1317722111

Lande, R., \& Arnold, S. J. (1983). The measurement of selection on correlated characters.

$600 \quad$ Evolution, 37(6), 1210. doi:10.2307/2408842

601 Laughlin, D. C. (2014). The intrinsic dimensionality of plant traits and its relevance to

602 community assembly. Journal of Ecology, 102(1), 186-193. doi:10.1111/1365-

$603 \quad 2745.12187$

604 Lauzeral, C., Leprieur, F., Beauchard, O., Duron, Q., Oberdorff, T., \& Brosse, S. (2011).

605 Identifying climatic niche shifts using coarse-grained occurrence data: a test with non-

606 native freshwater fish. Global Ecology and Biogeography, 20(3), 407-414.

607 doi:10.1111/j.1466-8238.2010.00611.x

608 Leimar, O., Sasaki, A., Doebeli, M., \& Dieckmann, U. (2013). Limiting similarity, species 609 packing, and the shape of competition kernels. Journal of Theoretical Biology, 339, 3-

$610 \quad$ 13. doi:10.1016/j.jtbi.2013.08.005

611 Lichstein, J. W. (2007). Multiple regression on distance matrices: A multivariate spatial 612 analysis tool. Plant Ecology, 188(2), 117-131. doi:10.1007/s11258-006-9126-3

613 Loiseau, N., Legras, G., Gaertner, J.-C., Verley, P., Chabanet, P., \& Mérigot, B. (2017).

614 Performance of partitioning functional beta-diversity indices: Influence of functional

615 representation and partitioning methods. Global Ecology and Biogeography, 26(6), 753-

$616 \quad$ 762. doi:10.1111/geb.12581

617 Lu, R. P., Smith, E. P., \& Good, I. J. (1989). Multivariate measures of similarity and niche

618 overlap. Theoretical Population Biology, 35(1), 1-21. doi:10.1016/0040-

$619 \quad 5809(89) 90007-5$

620 MacArthur, R., \& Levins, R. (1967). The limiting similarity, convergence, and divergence of 621 coexisting species. The American Naturalist, 101(921), 377-385. doi:10.1086/282505 
622 Mahony, C. R., Cannon, A. J., Wang, T., \& Aitken, S. N. (2017). A closer look at novel

623 climates: new methods and insights at continental to landscape scales. Global Change

624 Biology, 23(9), 3934-3955. doi:10.1111/gcb.13645

625 Mammola, S. (2019). Assessing similarity of $\mathrm{n} \square$ dimensional hypervolumes: Which metric

626 to use? Journal of Biogeography, 46(9), 2012-2023. doi:10.1111/jbi.13618

627 Mammola, S., \& Cardoso, P. (2020). Functional diversity metrics using kernel density n

$628 \square$ dimensional hypervolumes. Methods in Ecology and Evolution, 11(8), 986-995.

629 doi:10.1111/2041-210X.13424

630 Minami, M., \& Shimizu, K. (1999). Estimation of similarity measure for multivariate normal

631 distributions. Environmental and Ecological Statistics, 6(3), 229-248.

632 doi:10.1023/A:1009678412953

633 Morisita, M. (1959). Measuring of interspecific association and similarity between

634 communities. Memorandum, Faculty of Science, Kyushu Univ. Ser. E., 65-80.

635 doi:10.18960/seitai.11.6_252_4

636 Nunes, L. A., \& Pearson, R. G. (2017). A null biogeographical test for assessing ecological

637 niche evolution. Journal of Biogeography, 44(6), 1331-1343. doi:10.1111/jbi.12910

638 Pearman, P. B., Guisan, A., Broennimann, O., \& Randin, C. F. (2008). Niche dynamics in

639 space and time. Trends in Ecology and Evolution, 23(3), 149-158.

$640 \quad$ doi:10.1016/j.tree.2007.11.005

641 Pianka, E. R. (1974). Niche overlap and diffuse competition. Proceedings of the National

642 Academy of Sciences of the United States of America. doi:10.1073/pnas.71.5.2141

643 Pigot, A. L., Sheard, C., Miller, E. T., Bregman, T. P., Freeman, B. G., Roll, U., ... Tobias, J.

644 A. (2020). Macroevolutionary convergence connects morphological form to ecological

645 function in birds. Nature Ecology and Evolution, 4(2), 230-239. doi:10.1038/s41559-

$646 \quad 019-1070-4$ 
647 Pigot, A. L., Trisos, C. H., \& Tobias, J. A. (2016). Functional traits reveal the expansion and

648 packing of ecological niche space underlying an elevational diversity gradient in

649 passerine birds. Proceedings of the Royal Society B: Biological Sciences, 283(1822), 1-

650 9. doi:10.1098/rspb.2015.2013

651 Pironon, S., Villellas, J., Thuiller, W., Eckhart, V. M., Geber, M. A., Moeller, D. A., \&

652 García, M. B. (2018). The 'Hutchinsonian niche' as an assemblage of demographic

653 niches: implications for species geographic ranges. Ecography, 41(7), 1103-1113.

654 doi:10.1111/ecog.03414

655 Pulliam, H. R. (2000). On the relationship between niche and distribution. Ecology Letters,

656 3(4), 349-361. doi:10.1046/j.1461-0248.2000.00143.x

657 Rappoldt, C., \& Hogeweg, P. (1980). Niche Packing and Number of Species. The American

$658 \quad$ Naturalist, 116(4), 480-492. doi:10.1086/283643

659 Read, Q. D., Grady, J. M., Zarnetske, P. L., Record, S., Baiser, B., Belmaker, J., ... Thibault,

660 K. M. (2018). Among-species overlap in rodent body size distributions predicts species

661 richness along a temperature gradient. Ecography, 1-10. doi:10.1111/ecog.03641

662 Rolland, J., \& Salamin, N. (2016). Niche width impacts vertebrate diversification. Global

663 Ecology and Biogeography, 1252-1263. doi:10.1111/geb.12482

664 Sagarin, R. D., Gaines, S. D., \& Gaylord, B. (2006). Moving beyond assumptions to

665 understand abundance distributions across the ranges of species. Trends in Ecology and

666 Evolution, 21(9), 524-530. doi:10.1016/j.tree.2006.06.008

667 Saupe, E. E., Qiao, H., Hendricks, J. R., Portell, R. W., Hunter, S. J., Soberón, J., \&

668 Lieberman, B. S. (2015). Niche breadth and geographic range size as determinants of

669 species survival on geological time scales. Global Ecology and Biogeography, 24(10),

670 1159-1169. doi:10.1111/geb.12333

671 Serra-Varela, M. J., Grivet, D., Vincenot, L., Broennimann, O., Gonzalo-Jim??nez, J., \& 
672

673

674

675

676

677

678

679

680

681

682

683

684

685

686

687

688

689

690

691

692

693

694

695

696

Zimmermann, N. E. (2015). Does phylogeographical structure relate to climatic niche divergence? A test using maritime pine (Pinus pinasterAit.). Global Ecology and Biogeography, 24(11), 1302-1313. doi:10.1111/geb.12369

Slatyer, R. A., Hirst, M., \& Sexton, J. P. (2013). Niche breadth predicts geographical range size: A general ecological pattern. Ecology Letters, 16(8), 1104-1114. doi:10.1111/ele. 12140

Soberon, J., \& Nakamura, M. (2009). Niches and distributional areas: Concepts, methods, and assumptions. Proceedings of the National Academy of Sciences, 106(Supplement_2), 19644-19650. doi:10.1073/pnas.0901637106

Sullivan, B. L., Wood, C. L., Iliff, M. J., Bonney, R. E., Fink, D., \& Kelling, S. (2009). eBird: A citizen-based bird observation network in the biological sciences. Biological Conservation. doi:10.1016/j.biocon.2009.05.006

Swanson, H. K., Lysy, M., POwer, M., Stasko, A. D., Johnson, J. D., \& Reist, J. D. (2015). A new probabilistic method for quantifying n-dimensional ecological niches and niche overlap. Ecology, 96(2), 318-324. doi:10.1890/14-0235.1

Tayleur, C., Caplat, P., Massimino, D., Johnston, A., Jonzén, N., Smith, H. G., \& Lindström, Å. (2015). Swedish birds are tracking temperature but not rainfall: Evidence from a decade of abundance changes. Global Ecology and Biogeography, 24(7), 859-872. doi:10.1111/geb.12308

Whittaker, R. H., Levin, S. A., \& Root, R. B. (1973). Niche, Habitat, and Ecotope. The American Naturalist. doi:10.1086/282837

Wiens, J. J., Litvinenko, Y., Harris, L., \& Jezkova, T. (2019). Rapid niche shifts in introduced species can be a million times faster than changes among native species and ten times faster than climate change. Journal of Biogeography, 46(9), 2115-2125. doi:10.1111/jbi.13649 
697 Wilman, H., Belmaker, J., Simpson, J., de la Rosa, C., Rivadeneira, M. M., \& Jetz, W. (2014).

698 EltonTraits 1.0: Species-level foraging attributes of the world's birds and mammals.

$699 \quad$ Ecology, 95(7), 2027-2027. doi:10.1890/13-1917.1

700 Winner, K., Noonan, M. J., Fleming, C. H., Olson, K. A., Mueller, T., Sheldon, D., \&

701 Calabrese, J. M. (2018). Statistical inference for home range overlap. Methods in

702 Ecology and Evolution, 9(7), 1679-1691. doi:10.1111/2041-210X.13027

703 Zurell, D., Gallien, L., Graham, C. H., \& Zimmermann, N. E. (2018). Do long-distance

704 migratory birds track their niche through seasons? Journal of Biogeography, 45(7),

705 1459-1468. doi:10.1111/jbi.13351

706 